\title{
Heterogeneously integrated impedance measuring system with disposable thin-film electrodes
}

\author{
Hanbin Ma ${ }^{\mathrm{a}}$, Jiahao Li ${ }^{\mathrm{a}}$, Xiang Cheng ${ }^{\mathrm{a}}$, Arokia Nathan ${ }^{\mathrm{a}^{*}}$ \\ ${ }^{a}$ Electrical Division, Engineering Department, University of Cambridge, 9 JJ Thomson \\ Avenue, Cambridge, CB3 OFA, U.K. \\ * To whom correspondence should be addressed: Tel: +44 (0) 1223 748302, Fax: +44 (0) \\ 1223748322 \\ E-mail: an299@cam.ac.uk
}

\section{Keywords}

impedance biosensor, CMOS, active thin-film electrodes, DNA concentration, heterogeneous integration

\section{Highlights}

- An integrated impedance measuring system with disposable electrodes.

- Reusable CMOS chip for data retrieval from remote control the thin-film electrodes.

- We performed suspended DNA concentration tests.

- The system shows doubled sensitivity over previous work.

- Providing a new platform for integrated sensor with disposable interfaces. 


\begin{abstract}
We propose a novel integrated impedance measurement system with disposable thin-film electrodes. Most modern CMOS-based biosensors use on-chip electrodes to interface between the electronics and biosamples, which forces disposal of the CMOS chip after a few measurements, since most biological reactions are non-reversible. The sensor performance is also limited by the design of on-chip electrodes due to the physical dimensions and the CMOS design rules restrictions. In this work, we extract the electrodes from the silicon chip for relocation onto a low-cost, disposable substrate. This enables reusability of the high-performance CMOS chip, at the same time providing a low-cost route for manufacture of the active thin-film electrodes using large-area processing. The use of disposable thin-film chip also enables customized designed electrodes for different applications, such as extra high sensitivity concentration sensors. In this work, DNA concentration measurements are performed, and it shows a doubling of sensitivity over the previously reported system.
\end{abstract}




\section{Introduction}

Recent development of biosensing systems are being pushed towards high sensitivity, portability and low cost. For example, emerging technologies are enabling fluorescencebased detection to achieve high sensitivity and selectivity [1,2], and advanced luminescence assay can be specifically designed to fit different applications [3]. However, such performance requirements may not be necessary for most point-of-care (PoC) [4] and fast diagnostic applications where low cost and ease of operation are key design considerations. Indeed biosensors with compact and miniaturised design and moderate detection performance are potentially favourable candidates.

Electrochemical sensors, which detect and measure electrical signals instead of the fluorescence output in optical sensors, provides a label-free and rapid solution for biological detection [4,5]. They are now widely applied in detection of biomolecules from the macro to micro scale, such as cells [6], proteins [7] and DNA [8,9]. By taking advantage of CMOS technology, the performance of modern electrochemical sensors is significantly enhanced. High-performance systems can be integrated into a monolithic silicon chip, which is fast, low-cost and accurate. A variety of electrochemical methods based on CMOS chips have been developed. These include capacitance-based sensors [10], ion-sensitive field-effect transistor-based sensors [11,12] and impedance-based sensors $[13,14]$. Impedance measurement, which shows great promise for studying both the electrode-electrolyte interface and bulk solution, has been gaining popularity for biological sensing in recent years [15]. However, the development of CMOS-based solution for this application is limited by the on-chip electrodes. Most CMOS biosensors 
employ the top metal to create an electrode array reminiscent of the design principles used in CMOS image sensor arrays.

Unfortunately, top metal in a traditional CMOS process (e.g. copper) is not biocompatible, thus requiring post-CMOS fabrication process for deposition of additional bio-compatible metal layers on top of the silicon chip. Manickam et al. [14] reported the use of an electroless nickel immersion Au (ENIG) process, which deposits an adhesive nickel layer followed by a gold layer. Apart from the post-CMOS fabrication process, the on-chip electrode surfaces can be difficult to clean, and the residue of previous sample can affect the accuracy of subsequent measurement. This forces the disposal of the CMOS chip after a few measurements, which is not practical due to cost and environmental considerations. In addition, large-size electrodes are desirable in some applications such as cell level analysis [16,17]. Due to the integration of the on-chip electrodes, the cost of the CMOS chip can be significantly increased by increasing the silicon die area.

In this paper, we developed a heterogeneous impedance measurement system with a reusable CMOS chip for signal processing, and an active thin-film electrode disposable system as an electronics-biological interface. The disposable chip enables large-area fabrication technologies, which provide great freedom for the selection of electrode materials and electrode configurations. In addition, the design of the electrodes is separated from the CMOS chip, which can be customized for different applications. With the use of disposable electrodes, it can be used as a powerful platform for multifunctional impedance-based measurements. In this paper, the system performance was examined using suspended DNA samples in different concentrations. 


\section{Material and methods}

\subsection{The impedance sensing system}

Figure 1 shows a schematic of the integrated impedance measurement system. The system consists of two parts, including a disposable glass chip and a reusable part. Eight $\mathrm{Cr} / \mathrm{Au}(20 \mathrm{~nm} / 80 \mathrm{~nm})$ double layer metal electrodes were fabricated by a thermal evaporator with a shadow mask on a glass substrate. Both the width of each electrode and the separation between the electrodes are $500 \mu \mathrm{m}$. After the metal deposition, the electrodes were then attached to connection pins by silver paste. A 5 mm diameter metal cap was used to cover the centre part of the electrodes. The rest of the glass chip was covered by liquid PDMS to passivate and hold the connection pins. After the PDMS solidified, the cap was removed to leave a $5 \mathrm{~mm}$ diameter well on top of the electrodes. This well was used to contain the sample solution during the measurement. The fabrication of the electrode chip is low-cost and highly reproducible. The glass chip is the interface between sensor and the sample, which can be disposed after each measurement.

The impedance measurement chip was designed in a Cadence analogue IC design suite, and the prototype was fabricated using AMS 0.18um CMOS process. The design was an extended work to our previous impedance-based CMOS chip, but here sensing electrodes are extracted from the silicon chip on to a disposable glass substrate.

A complete measurement system is shown in Fig. 1. The CMOS chip is mounted on a PCB and connected to a disposable sensor via Dupont wires. The excitation signals applied to the electrodes on a disposable sensor and lock-in amplifier in CMOS chip are provided by dual-channel function generator. The voltage signal from the CMOS IC is 
further filtered and readout from oscilloscope. A detailed CMOS design plan can be found in Appendix A.

Although commercial ICs for electrochemical impedance measurement are available in the market, they are only suited to sensors with general purpose specifications. Customdesigned ICs provide the needed freedom to circuit designers for more specific requirements given by a certain sensor type.

\subsection{System validation}

A simple impedance circuit with three discrete elements was built to validate the performance of the impedance chip. As shown in Fig. 2(a) subplot, a $10 \mathrm{k} \Omega$ resistor $\left(R_{2}\right)$ in parallel with a $1 \mathrm{nF}$ capacitor $\left(C_{1}\right)$ is connected to a $1 \mathrm{k} \Omega$ resistor $\left(R_{1}\right)$. The tolerances of metal film resistors and ceramic capacitor are $10 \%$ and $20 \%$ respectively. The elements were mounted by the standard soldering technique. Ideal values were used for post-layout simulation by the Cadence Virtuoso software. Measurements with the threeelement circuit and CMOS chip were carried out from $100 \mathrm{~Hz}$ to $10 \mathrm{MHz}$.

\subsection{Preparation of DNA samples with different concentrations}

Herring sperm DNA was ordered from SIGMA-ALDRICH (D7290). The single-stranded DNA fragments ranged in size from 587 to 831 base pairs. This DNA was provided as a ready-to-use concentrated solution (9-12 mg/ml DNA). According to the supplier's instructions, we boiled the solution for 10 minutes and then cooled it on ice for another 10 minutes to reduce the likelihood of re-annealing the fragments. We first diluted $20 \mu \mathrm{l}$ of the concentrated DNA sample with $180 \mu \mathrm{l}$ of DI water to prepare the stock solution. 
The concentration of the stock solution was determined to be $1.2 \mathrm{mg} / \mathrm{ml}(1200 \mu \mathrm{g} / \mathrm{ml})$ using a Nanodrop ${ }^{\mathrm{TM}}$ 2000. We diluted the stock solution with DI water to give different concentrations:

- $100 \mu \mathrm{l}$ of stock solution and $100 \mu \mathrm{l}$ of DI water for a concentration of $600 \mu \mathrm{g} / \mathrm{ml}$;

- $100 \mu \mathrm{l}$ of $600 \mu \mathrm{g} / \mathrm{ml}$ solution and $100 \mu \mathrm{l}$ of DI water for a concentration of 300 $\mu \mathrm{g} / \mathrm{ml}$;

- $100 \mu \mathrm{l}$ of $300 \mu \mathrm{g} / \mathrm{ml}$ solution and $100 \mu \mathrm{l}$ of DI water for a concentration of 150 $\mu \mathrm{g} / \mathrm{ml}$;

- $100 \mu \mathrm{l}$ of $150 \mu \mathrm{g} / \mathrm{ml}$ solution and $100 \mu \mathrm{l}$ of DI water for a concentration of $75 \mu \mathrm{g} /$ $\mathrm{ml}$;

- $\quad 100 \mu \mathrm{l}$ of $75 \mu \mathrm{g} / \mathrm{ml}$ solution and $100 \mu \mathrm{l}$ of DI water for a concentration of 37.5 $\mu \mathrm{g} / \mathrm{ml}$.

The concentrations of all diluted samples were validated using commercial spectrophotometers Nanodrop. All of the sample preparation steps were performed at room temperature, and $60 \mu \mathrm{l}$ of sample was used for each measurement.

\subsection{Equivalent circuit fitting for impedance measurement}

All the impedance measurement data were fitted by the commercial software ZView ${ }^{\circledR}$, supplied by Scribner Associated Inc. The standard Randles circuit model (shown in Fig. 4(a) subplot) was used for DNA sample fitting.

\section{Results and discussion}

\subsection{System validation test}


Figure 2 shows the Bode plots of three-element circuit, post-layout simulation and measured results of the validation tests. The data indicated in red is the ideal frequency response of the three-element model shown in subplot in Fig. 2(a), and the corresponding component values are labelled as well. At lower frequencies, the capacitor $\left(C_{1}\right)$ was treated as open circuit, and the total impedance was equal to the sum of $R_{1}$ and $R_{2}$. As the frequency increases, the circuit current starts to leak from the capacitor. The impedance of the capacitor decreases, and as a result, the impedance of the three-element model decreases. At higher frequencies, the capacitor $\left(C_{1}\right)$ shorts the resistor $\left(R_{2}\right)$, and the total impedance decreases to the lowest point, and equals to the value of $R_{1}$. The negative phase between $50 \mathrm{kHz}$ to $500 \mathrm{kHz}$ indicates a signal delay due to the capacitance. Note that there is no positive phase data in the ideal response as there is no inductive element in the circuit.

The green data line depicts post layout simulation results of the CMOS chip with the three-element circuit as elements-under-test. The key components of the impedance chip were amplifiers, which helped amplify the sensing current signal and converting it into a voltage. Assuming an AC stimulus, a voltage $V_{\text {in }}$ was applied to the elements-under-test. The resulting current $I_{\text {sense }}$ was sensed and converted into a voltage output $V_{\text {out }}$ through several stages with a total gain $K$. The impedance magnitude $(|Z|)$ of the elements-undertest can be stated as

$|Z|=\frac{V_{\text {in }}}{I_{\text {sense }}}=\frac{V_{\text {in }}}{\frac{V_{\text {out }}}{K}}=\frac{V_{\text {in }} \cdot K_{0}}{V_{\text {out }}}$

where $K_{0}$ represents the constant system gain over the low frequency band. The simulated results follow the ideal data closely in this region. As the frequency increases, the total 
gain starts to roll-off, and the system cut-off frequency is about $1 \mathrm{MHz}$. The actual system gain is smaller than the value of $K_{0}$ used in Equation (1), therefore, the calculated impedance $(|Z|)$ is greater. From the comparison of the ideal response and simulation, we are able to determine that most reliable frequency region for system operation is below 1 $\mathrm{MHz}$.

Two discrete resistors and a capacitor were used in measurements. The three-element circuit was connected to the PCB directly and the measured results indicated in blue are shown in Fig. 2. The measured impedance closely follows simulation results over all frequencies, validating the use of the measurement technique to evaluate the impedance of the sample-under-test.

The measured result of the three-element circuit has also been fitted by the three-element model, and the results are shown in Fig. 3. The fitted element values are also shown in the subplot in Fig. 3(a). For $R_{2}$ and $C_{2}$, the fitted values are similar to the element values with a discrepancy less than $5 \%$. Note that, the fitted value of $R_{1}$ is $700 \Omega$ greater than the nominated value. This is due to the interconnections between the circuit elements and the PCB and related contact resistance issue. This is one of the major drawbacks of remote electrode measurements, as the undesired parasitic impedance contributes to the overall system error. In this work, silver paste and Dupont wires were used for the interconnection between glass chip and PCB, which is not ideal and provides large undesirable parasitic. This can be improved by other connection techniques in future work, such as anisotropic conductive film bonder. According to the previous research, a typical DNA solution impedance is around hundreds kilo-ohm level. In our system, the parasitic impedance is around $700 \Omega$, and it only contributes to less than $1 \%$ discrepancy. 


\subsection{DNA concentration test}

The DNA sample concentration tests were performed with the CMOS chip and external thin-film electrodes, as described in Section 2. Five groups of herring sperm DNA samples with different concentrations were characterized. The measurements were carried out at frequencies from $100 \mathrm{~Hz}$ to $1 \mathrm{MHz}$. Figure 4 shows the results in both Cole-Cole and Bode plots. The former yields the general impedance behaviour of the samples. At low DNA concentrations $(37.5 \mu \mathrm{g} / \mathrm{ml}, 75 \mu \mathrm{g} / \mathrm{ml}$ and $150 \mu \mathrm{g} / \mathrm{ml})$, each dataset forms a semicircle with a tail, which is a classic pattern for bio-solutions [19]. The semicircle is formed by the double layer capacitance between the electrodeelectrolyte interfaces. The DNA solutions were prepared using DI water and hence the main charge carriers within the solution are the DNA with each base pair contained two negative charges. Higher concentration DNA meant more base pairs per volume and hence, more charges per unit volume. Therefore, the concentrated DNA solutions were much conductive and as the solution concentrates, the radius of the semicircle starts to shrink. This indicates that highly conductive solutions are more resistive than capacitive. As the Cole-Cole plot shown, a purely resistive sample manifests as a straight line parallel to the imaginary axis, which implies that the complex impedance only contains the real part. This is particularly true for the most concentrated sample (600 $\mu \mathrm{g} / \mathrm{ml})$, which comes up a straight line and is almost parallel to the imaginary axis.

The Bode plot shows the magnitude and the phase of the impedance for different samples. The impedance values are well spread over the frequency range from $100 \mathrm{~Hz}$ to $1 \mathrm{MHz}$, which indicates the clear differences in electrical properties of the samples. Each set of impedance data was fitted to a same equivalent circuit, and the results are shown in grey 
lines. The Randles circuit model was selected in this work, which is the classic model for depicting the electrode-electrolyte interface and the bulk solution. Figure 4 clearly shows the good agreement between the fitted results and five group samples. The fitting parameters can be found in the Appendix B. The only mismatches are at the high frequency end for higher concentration samples. The measured data shows a positive phase error, which is not practical in solution-based impedance measurements. This is stress from the parasites, such as stray capacitance and contact resistance.

From the Bode plot shown in Fig. 4, the magnitude of impedance for each DNA sample is relatively constant at mid-band frequencies $(1 \mathrm{kHz}$ to $100 \mathrm{kHz})$. Higher sample concentration results lower sample impedance.

Figure 5 depicts comparison results of this work and previous work for DNA concentration measurement. The detection sensitivities can be therefore determined by the slopes of two data lines. From the least linear square fitting results, this work shows almost doubled sensitivity over the previous work, where the slope of this work is 0.85 and the previous one is 0.44 . The specific thin-film electrodes design in this work contributes to this sensitivity enhancement.

In a DC electrolyte sensing system, cell constant $\kappa\left(\mathrm{cm}^{-1}\right)$ is defined as

$\kappa=\frac{R}{\rho}$

where $R$ represents the measured resistance and $\rho$ is the specific resistivity. This factor also can be used to determine the performance of an impedance-based sensing system. For most bio-measurement, the solution concentration $(c)$ is a function of the solution 
conductivity (reciprocal of the solution resistivity). From Equation (1), we can conclude that the sensing output voltage $\left(V_{\text {out }}\right)$ is inversely proportional to the measured impedance. We can then rewrite Equation (2) as

$\frac{V_{\text {out }}}{c} \propto \frac{1}{\kappa}$

where $c$ represents the solution concentration. Equation (3) indicates that smaller cell constant will result better detection sensitivity, therefore, to design a small cell constant system is a key route to enhance the sensor's performance. Olthuis’s work [20] suggests that the cell constant can be reduced effectively by increasing the electrodes modulus $k$. In a two electrodes system, $k$ is defined as

$k=\frac{W}{W+S}$

where $W$ is the electrode width and $S$ is the separation between the electrodes pair. Thus, a better $\mathrm{W}$ over $\mathrm{S}$ ratio can give better detection sensitivity. The $W$ over $S$ ratio is 1:2 in previous work (shown as green in Fig. 5), and the ratio is 1:1 in this work (shown as blue). In previous work, on-chip electrodes are used, and the electrodes dimensions are limited by both chip area and CMOS design rules. However, by using thin-film based electrodes, the electrodes design is more flexible and hence the sensing performance can be further enhanced. Further analysis regarding $W$ over $S$ ratio can be found in Appendix C.

It is worth to mention that bare gold electrodes are used in this work for characterisation of suspended DNA samples. This is different from reported affinity-based sensors in which electrodes are functionalised with probes to capture target analytes. Immobilised probes are typically used to examine the presence of known analytes. However, both 
limit of detection and accuracy are restricted by unspecific binding and binding failure. In our work, the electrical properties of the bulk solution are measured from $100 \mathrm{~Hz}$ to 1 MHz. This method can be used as an effective DNA concentration sensor. It also provides the capabilities to distinguish the length of DNA fragments, and can be applied to validate PCR products. Indeed, sensors without immobilisation lack selectivity compared to the conventional affinity-based approaches. However, this can be solved by introducing micro-fluidic sample sorting and/or sieving technique on top of electrodes in the future work. Moreover, this heterogeneously measuring system is fully compatible with bio-functionalization on the electrodes, which can be used for different specific applications.

\section{Conclusions}

A heterogeneously integrated impedance measuring system is presented with disposable thin-film electrodes. The system keeps the CMOS processor reusable in order to reduce the measurement cost. This setup also provides additional freedom on the electrode design. For DNA concentration measurement, this system enables a doubled sensitivity measurement over the previous work.

\section{Acknowledgements}

This work is partially supported by Isaac Newton Trust.

\section{Reference}

[1] N. Sui, L. Wang, T. Yan, F. Liu, J. Sui, Y. Jiang, Selective and sensitive biosensors based on metal-enhanced fluorescence, Sensors Actuators B Chem. 202 (2014) 1148-1153. 
[2] H. Dai, Y. Shi, Y. Wang, Y. Sun, J. Hu, P. Ni, A carbon dot based biosensor for melamine detection by fluorescence resonance energy transfer, Sensors Actuators B Chem. 202 (2014) 201-208.

[3] G. Lautner, Z. Balogh, A. Gyurkovics, R.E. Gyurcsányi, T. Mészáros, Homogeneous assay for evaluation of aptamer-protein interaction., Analyst. 137 (2012) 3929-31.

[4] J. Wang, Electrochemical biosensors: towards point-of-care cancer diagnostics., Biosens. Bioelectron. 21 (2006) 1887-92.

[5] R.E. Gyurcsányi, T. Vigassy, E. Pretsch, Biorecognition-modulated ion fluxes through functionalized gold nanotubules as a novel label-free biosensing approach., Chem. Commun. (2003) 2560-1.

[6] M. Varshney, Y. Li, Interdigitated array microelectrodes based impedance biosensors for detection of bacterial cells., Biosens. Bioelectron. 24 (2009) 295160 .

[7] P. Kara, A. de la Escosura-Muñiz, M. Maltez-da Costa, M. Guix, M. Ozsoz, A. Merkoçi, Aptamers based electrochemical biosensor for protein detection using carbon nanotubes platforms., Biosens. Bioelectron. 26 (2010) 1715-8.

[8] T.G. Drummond, M.G. Hill, J.K. Barton, Electrochemical DNA sensors., Nat. Biotechnol. 21 (2003) 1192-9.

[9] H. Ma, R.W.R. Wallbank, R. Chaji, J. Li, Y. Suzuki, C. Jiggins, A. Nathan, An impedance-based integrated biosensor for suspended DNA characterization, Sci. Rep. 3 (2013) 2730.

[10] K. Lee, S. Choi, J. Lee, CMOS capacitive biosensor with enhanced sensitivity for label-free DNA detection, Solid-State Circuits, IEEE, (2012) 120-122.

[11] J. Bausells, J. Carrabina, a Errachid, a Merlos, Ion-sensitive field-effect transistors fabricated in a commercial CMOS technology, Sensors Actuators B Chem. 57 (1999) 56-62.

[12] W. Chan, An integrated isfets instrumentation system in standard CMOS technology, Solid-State Circuits, IEEE, 45 (2010) 1923-1934.

[13] F. Heer, W. Franks, a Blau, S. Taschini, C. Ziegler, a Hierlemann, CMOS microelectrode array for the monitoring of electrogenic cells., Biosens. Bioelectron. 20 (2004) 358-66. 
[14] A. Manickam, A. Chevalier, M. McDermott, A.D. Ellington, A. Hassibi, A CMOS Electrochemical Impedance Spectroscopy (EIS) Biosensor Array., IEEE Trans.

Biomed. Circuits Syst. 4 (2010) 379-90.

[15] S. Park, J.-S. Yoo, With impedance data, a complete description of an electrochemical system is possible., Anal. Chem. (2003) 455-461.

[16] Q. Liu, J. Yu, L. Xiao, J.C.O. Tang, Y. Zhang, P. Wang, et al., Impedance studies of bio-behavior and chemosensitivity of cancer cells by micro-electrode arrays., Biosens. Bioelectron. 24 (2009) 1305-10.

[17] P. Brito, A.P.F. Turner, Mediated Biocatalytic Electrodes and Enzyme Stabilisation for Power Generation, Electroanalysis. 22 (2010) 732-743.

[18] M. Sun, S. Pejanovic, J. Mijovic, Dynamics of deoxyribonucleic acid solutions as studied by dielectric relaxation spectroscopy and dynamic mechanical spectroscopy, Macromolecules. (2005) 9854-9864.

[19] A.J. Bard, L.R. Faulkner, Electrochemical methods: fundamentals and applications, Wiley New York, 1980.

[20] W. Olthuis, W. Streekstra, P. Bergveld, Theoretical and experimental determination of cell constants of planar-interdigitated electrolyte conductivity sensors, Sensors Actuators B Chem. 25 (1995) 252-256. 


\section{Figures}

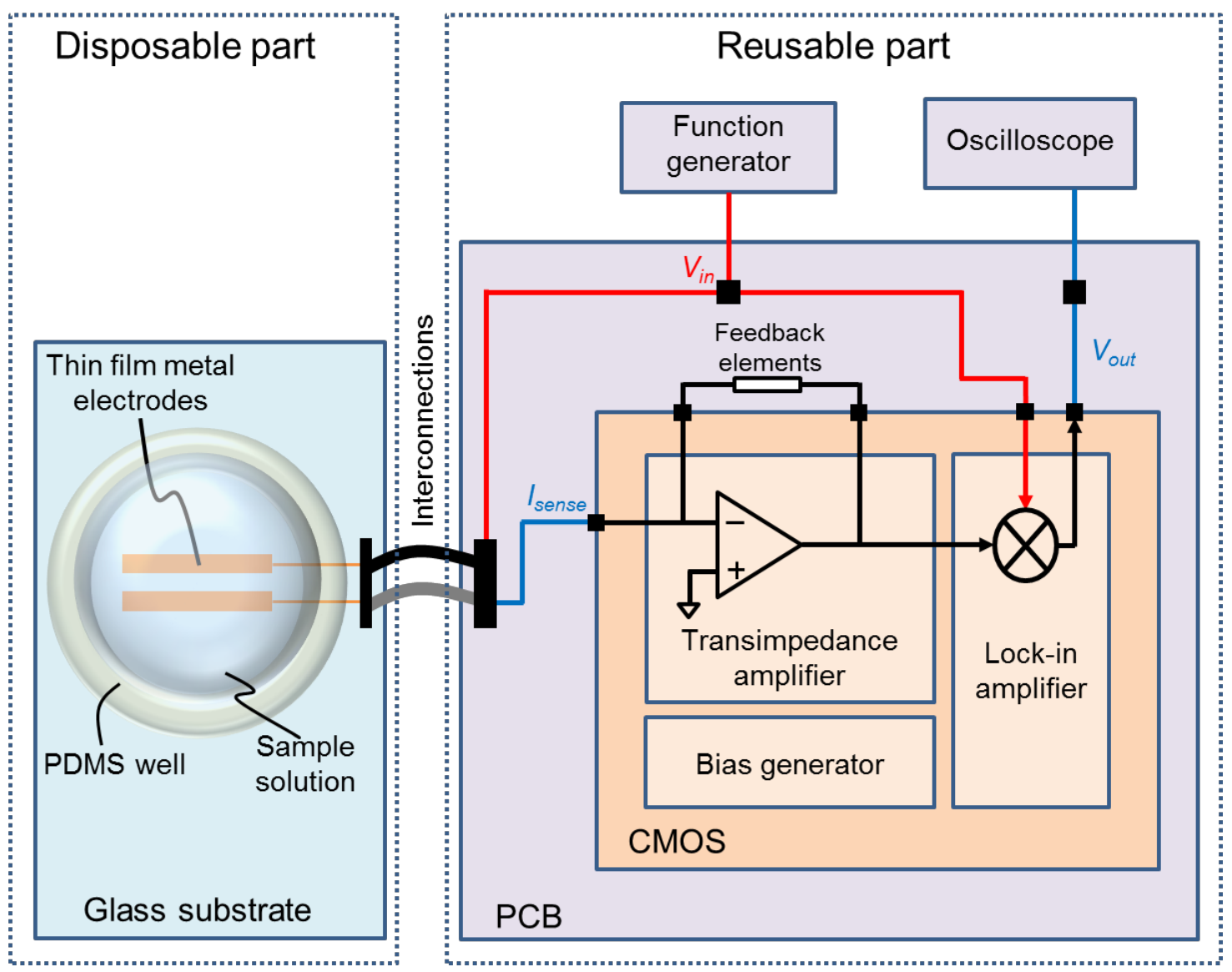

Fig. 1. Schematic of heterogeneously integrated impedance measurement system. The disposable part is thin-film metal electrodes on the glass substrate and reusable part includes a function generator for stimulus, a CMOS chip for signal amplification and processing and an oscilloscope. 

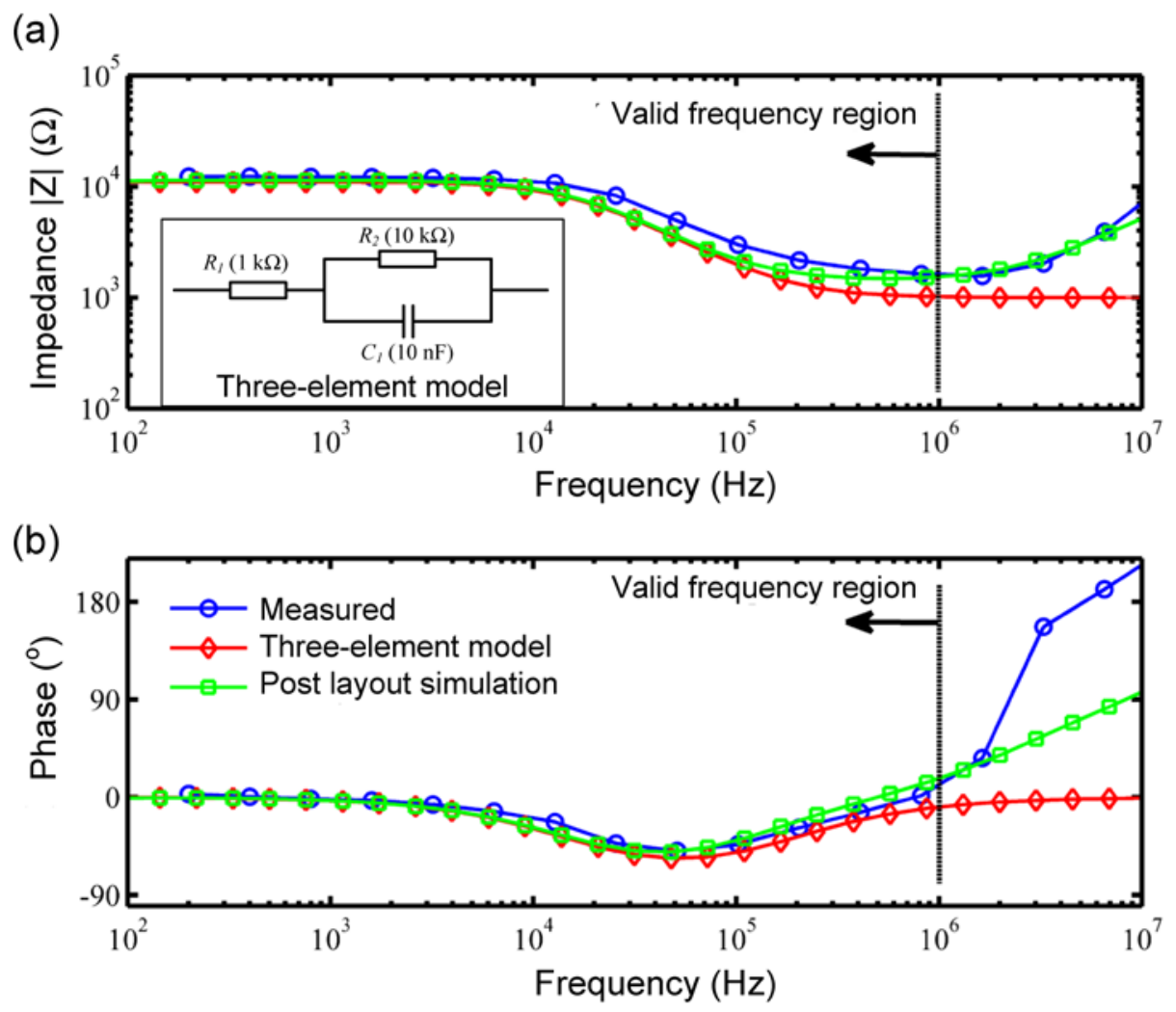

Fig. 2. Bode plot of simulation and measured results of the validation test for the frequency. (a) Magnitude of impedance as a function of frequency. Inset shows a threeelement circuit model and component values used for simulation. (b) As in (a) but phase as a function of frequency. 
(a)

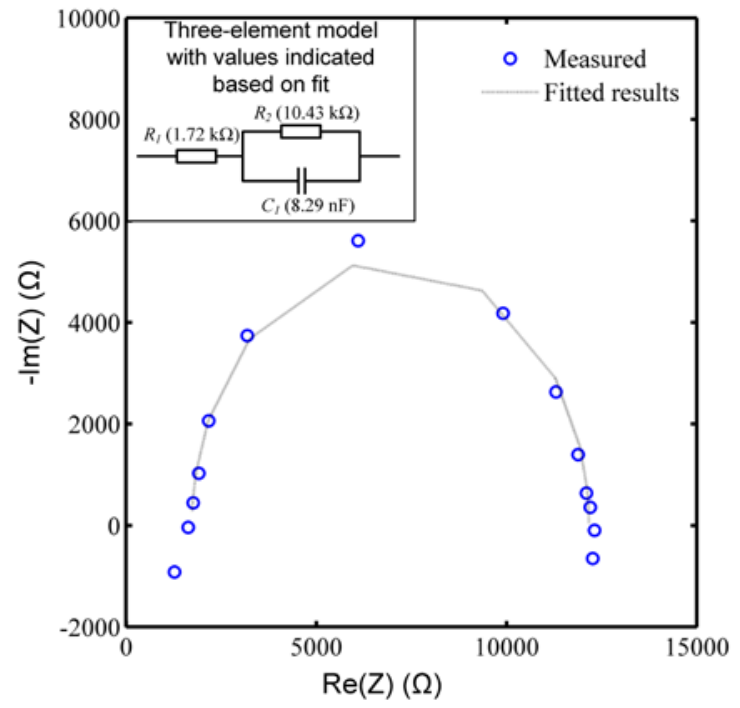

(b)

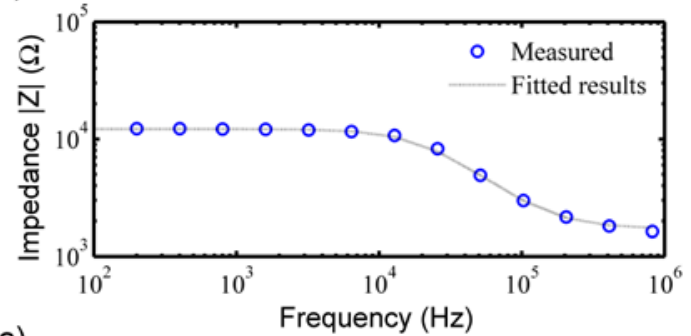

(c)

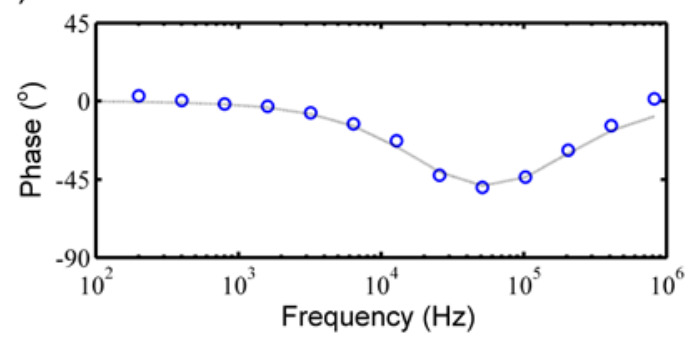

Fig. 3. (a) Cole-Cole plot of the measured and fitted results using a three-element circuit model; circuit model is indicated as inset. (b) (c) Bode plot of the measured and fitted results of the three-element circuit model shown in (a).

(a)

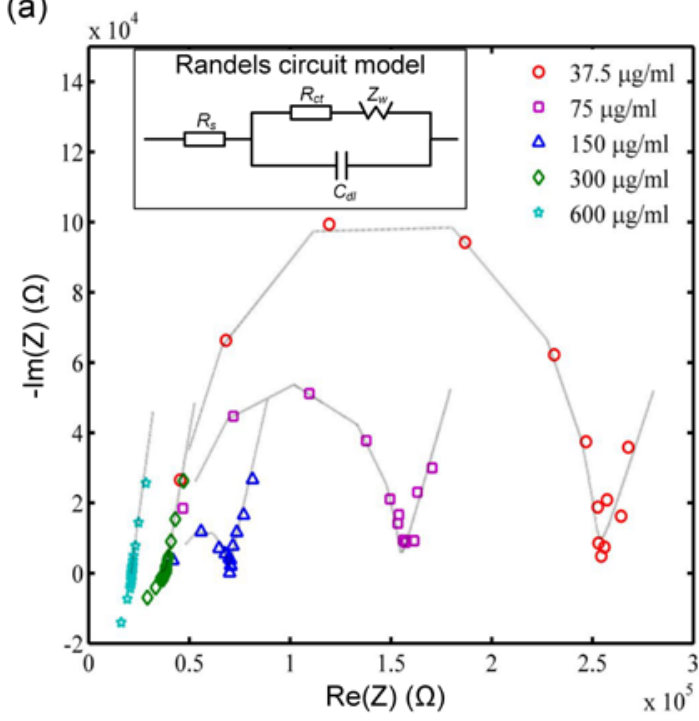

(b)
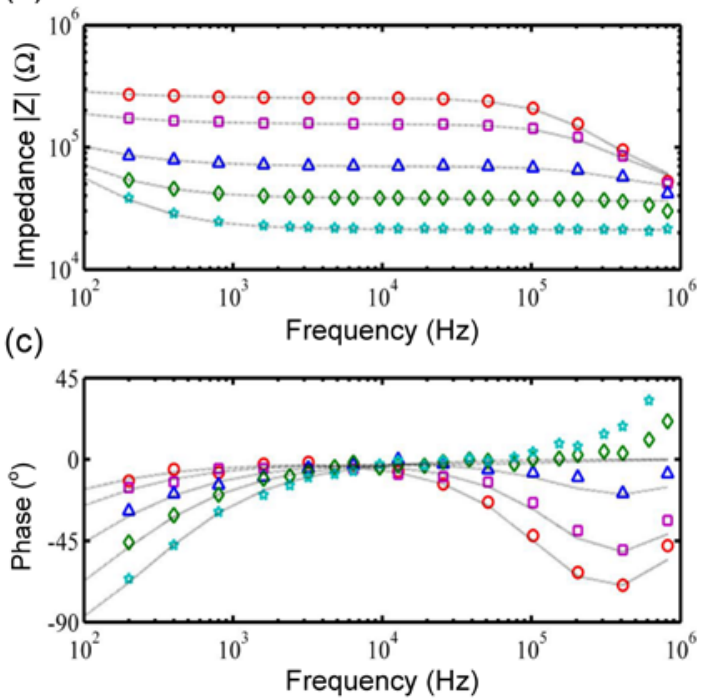

Fig. 4. (a) Cole-Cole plot of the measured and fitted results of different DNA concentrations. Inset shows a Randels circuit model used for data fitting. (b)(c) Bode plot of the measured and fitted values from (a). 


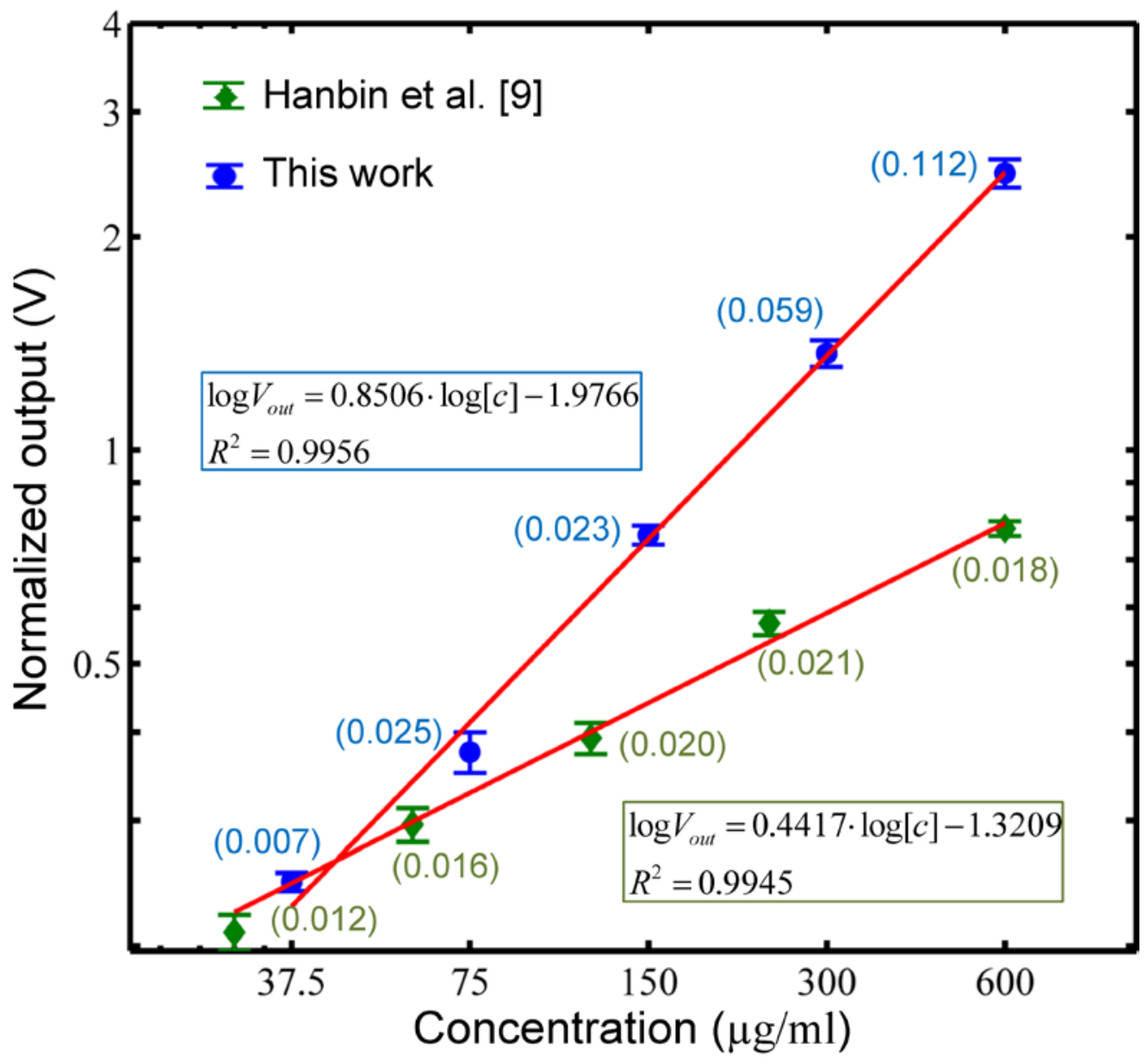

Fig. 5. DNA concentration versus normalized output with standard deviation indicated as error-bar. The slopes indicate sensitivity; this work shows an enhanced sensitivity for DNA concentration compared to previous work [9]. 